\title{
Applied LPV Control with Full Block Multipliers and Regional Pole Placement
}

\author{
Alejandro S. Ghersin ${ }^{1}$ and Ricardo S. Sánchez Peña ${ }^{2}$ \\ ${ }^{1}$ Departamento de Ingeniería Electrónica, Instituto Tecnológico de Buenos Aires (ITBA), Avenida Eduardo Madero 399, \\ C1106ACD, Buenos Aires, Argentina \\ ${ }^{2}$ Consejo Nacional de Investigaciones Científicas y Técnicas (CONICET) and Centro de Sistemas y Control - ITBA, Argentina
}

Correspondence should be addressed to Alejandro S. Ghersin, aghersin@itba.edu.ar

Received 1 July 2009; Revised 27 November 2009; Accepted 9 February 2010

Academic Editor: Venkataramanan Balakrishnan

Copyright ( 2010 A. S. Ghersin and R. S. Sánchez Peña. This is an open access article distributed under the Creative Commons Attribution License, which permits unrestricted use, distribution, and reproduction in any medium, provided the original work is properly cited.

\begin{abstract}
A formulation of an LPV control problem with regional pole placement constraints is presented, which is suitable for the application of a Full Block S-Procedure. It is demonstrated that improved bounds can be obtained on the induced $L_{2}$ norm of closed loop systems, while satisfying pole placement constraints. An application consisting in the 6 degrees of freedom (DOF) control of a space vehicle is developed as an example, with hardware in the loop (HIL) simulation. This shows that the method is appealing from the practical point of view, considering that the synthesized control law can be implemented satisfactorily in standard flight control systems. Conclusions with remarks towards the practical use of the method are presented as well.
\end{abstract}

\section{Introduction}

An interesting technique that has allowed linear parameter varying (LPV) control synthesis algorithms to obtain less conservative performance bounds is given the name of Full Block S-Procedure (FBSP). See $[1,2]$ and references therein. The formulas in $[3,4]$, render synthesis conditions combining the techniques of $[1,5]$, to develop a generalized framework for LPV control, based upon parameter dependent Lyapunov functions (PDLFs) and full block multipliers (FBMs). Yet these results provide linear matrix inequality (LMI) constrains with an infinite number of inequalities. In practice, one must resort to gridding the parameter variation set in order to apply the methods.

Subsequent work presented in [6] makes focus in particular form of PDLFs, namely, PDFLs that depend on the parameter in a linear fractional fashion, for systems whose open loop state space matrices depend on the parameter in a linear fractional way as well. In the sequel we will call the former LFT PDLFs and the latter LFT systems. From the practical point of view, the technique, which is based upon [4], is most appealing considering renders a set of constraints with a finite number of LMIs.
Another reference that is relevant from the practical point of view in LPV control is given by [7]. This work compares the results of [5] with an extension to LPV systems of the results in [8]. Helpful hints are given, in order to obtain LPV controllers that can be implemented in practice. The synthesis methods seek to establish stability and performance making use of PDLFs.

It must be recalled that synthesis conditions like the ones presented in [5] require a first step in order to establish feasibility of the problems and a second step to calculate the controllers. Under certain rank restrictions imposed on the state space matrices of the augmented plant, closed form expressions can be given for the calculation of the controllers. As opposed to this case, in [8] like results, an optimization is directly carried out, on a set of variables which are equivalent to the final state space matrices of the controller, through a nonlinear change of variables, which is the approach followed in this work.

It is rather frequent, in the application of LPV methods, that feasible problems with acceptable $\gamma$-performance indexes (see $[5,9]$ for a definition) show undesirable transient response. With a slight abuse of terminology, it can be posed as LPV systems having closed loop "poles" 
in undesirable locations. It is actually the poles of each linear time invariant system (LTI) resulting from holding the parameter vector constant, that turn out to have a nonconvenient loci. It has been reported previously that LPV synthesis, in particular when approached through a single quadratic Lyapunov function (SQLF), shows a problem called "fast poles" (see [10]).

The aspect of transient response of systems has not been dealt with in the FBSP framework of [6]. The work presented here extends the application of the FBSP, together with LFT PDLFs, to the same kind of LFT systems [6] deals with. The proposed approach includes the possibility of having the closed loop poles of each LTI system resulting from LPV dynamics with constant parameter trajectories, to have their loci in a prescribed region. This idea is most appealing from the practical viewpoint, in order to carry out the simulation and implementation of controllers. An application to the problem of 6 degrees of freedom (DOF) control of a spacecraft is presented with numerical hardware in the loop (HIL) simulations, as an application example.

The paper is organized as follows. Section 2 presents the developed formulas for LPV control with closed loop regional pole placement and FBMs. In Section 3, the synthesis method is employed to design the 6 DOF control for a rocket. Concluding remarks are given in Section 4 .

\section{Synthesis Method}

In this section the synthesis method of LPV control with FBMs, PDLFs, and regional pole placement constraints is presented.

2.1. Background. The set $\mathcal{P} \subset \mathbb{R}^{s}$ is such that for each $\theta=\left(\theta_{1}, \ldots, \theta_{s}\right) \in \mathcal{P},\left|\theta_{i}\right| \leq 1$. On the other hand, for some $\bar{\nu}=\left(\bar{\nu}_{1}, \ldots, \bar{\nu}_{s}\right) \in \mathbb{R}^{s}$ with $\bar{\nu}_{i}>0$, all $\nu=\left(\nu_{1}, \ldots, v_{s}\right) \in$ $\mathcal{V} \subset \mathbb{R}^{s}$ are such that $\left|\nu_{i}\right| \leq \bar{\nu}_{i}$. An $r_{\alpha}=\left(r_{\alpha_{1}}, \ldots, r_{\alpha_{s}}\right) \in \mathbb{N}^{s}$ defines the sets $\Theta_{\alpha}=\left\{\Theta_{\alpha}=\operatorname{diag}\left\{\theta_{1} I_{r_{\alpha_{1}}}, \ldots, \theta_{s} I_{r_{\alpha_{s}}}\right\}: \theta \in\right.$ $\mathcal{P}\}$ and $\dot{\boldsymbol{\Theta}}_{\alpha}=\left\{\dot{\Theta}_{\alpha}=\operatorname{diag}\left\{\dot{\theta}_{1} I_{r_{\alpha_{1}}}, \ldots, \dot{\theta}_{s} I_{r_{\alpha_{s}}}\right\}: \dot{\theta} \in \mathcal{V}\right\}$. The number $n_{\alpha}:=\sum_{i=1}^{s} r_{\alpha_{i}}$ is used later. In the sequel, any $r_{\beta}=\left(r_{\beta_{1}}, \ldots, r_{\beta_{s}}\right) \in \mathbb{N}^{s}$ will be regarded as defining a number $n_{\beta}$ and a couple of sets $\boldsymbol{\Theta}_{\beta}$, and $\dot{\boldsymbol{\Theta}}_{\beta}$, in the same fashion as $n_{\alpha}, \boldsymbol{\Theta}_{\alpha}$, and $\dot{\boldsymbol{\Theta}}_{\alpha}$ before. With the subindex omitted, it will be just $r=\left(r_{1}, \ldots, r_{s}\right) \in \mathbb{N}^{s}$, defining $n_{p}:=\sum_{i=1}^{s} r_{i}, \boldsymbol{\Theta}$ and $\dot{\boldsymbol{\Theta}}$. Throughout this paper, systems state space matrices depend on $s$-dimensional parameter trajectories evolving in the set $\mathcal{F}_{\mathcal{P}}^{\nu}=\left\{\theta \in \mathcal{C}^{1}\left(\mathbb{R}+, \mathbb{R}^{s}\right): \theta(t) \in \mathcal{P}, \dot{\theta}(t) \in \mathcal{V}\right.$, for all $\left.t \in \mathbb{R}_{+}\right\}$.

The following lemma is crucial in order to use FBMs for LPV control.

Lemma 1 (Full Block S-Procedure). Let

$$
\mathcal{g}(\Theta)=\Theta \star\left[\begin{array}{l|l}
G_{11} & G_{12} \\
\hline G_{21} & G_{22}
\end{array}\right]:=G_{22}+G_{21} \Theta\left(I-G_{11} \Theta\right)^{-1} G_{12}
$$

be a linear fractional transformation (LFT) where $G_{11}, G_{12}$, $G_{21}$ and $G_{22}$ are real matrices of compatible dimensions. Given a real symmetric matrix $M$, the quadratic matrix inequality

$$
g^{T}(\Theta) M g(\Theta)<0
$$

holds for all $\Theta \in \Theta$, if and only if there exists a real symmetric full-block multiplier $\Pi$ such that for any $\Theta \in \Theta$,

$$
\left[\begin{array}{c}
* \\
*
\end{array}\right]^{T} \operatorname{diag}\{\Pi, M\}\left[\begin{array}{cc}
G_{11} & G_{12} \\
I & 0 \\
\hline G_{21} & G_{22}
\end{array}\right]<0, \quad\left[\begin{array}{l}
I \\
\Theta
\end{array}\right]^{T} \Pi\left[\begin{array}{l}
I \\
\Theta
\end{array}\right] \geq 0 .
$$

Proof. See [6].

Remark 1. Condition (3) consists of an infinite number of constraints. Considering $\Theta$ a compact set defined by its $2^{s}$ vertices, additional constraints can be added in order to turn (3) into a condition with a finite number of constraints. Namely, partition the multiplier $\Pi$ as

$$
\Pi=\left[\begin{array}{ll}
\Pi_{11} & \Pi_{12} \\
\Pi_{12}^{T} & \Pi_{22}
\end{array}\right],
$$

and request $\Pi_{22}<0$. Then condition (3) will be convex with respect to $\Theta$. As a consequence, if for all vertices $\Theta_{i}$ of $\Theta$, the following LMI constraints

$$
\left[\begin{array}{c}
I \\
\Theta_{i}
\end{array}\right]^{T}\left[\begin{array}{ll}
\Pi_{11} & \Pi_{12} \\
\Pi_{12}^{T} & \Pi_{22}
\end{array}\right]\left[\begin{array}{c}
I \\
\Theta_{i}
\end{array}\right] \geq 0
$$

are satisfied, then inequality (3) will be so itself. This remark is important from the computational point of view. Notice that, while acceptable in practice, the approach can be conservative. Moreover, as observed in [6], fulfillment of constraint (3) is achieved if it is further enforced that $\Pi_{11}=-\Pi_{22}>0$, with $\Pi_{11}, \Pi_{22}$ being symmetric, $\Pi_{12}$ being skew-symmetric, and all of them being commutable with all $\Theta$ in $\Theta$.

In order to state the control problem, consider an LFT parameter-dependent plant:

$$
\left[\begin{array}{l}
\dot{x}(t) \\
e(t) \\
y(t)
\end{array}\right]=\underbrace{\left[\begin{array}{lll}
\mathcal{A}(\Theta(t)) & \mathcal{B}_{1}(\Theta(t)) & \mathcal{B}_{2}(\Theta(t)) \\
\mathcal{C}_{1}(\Theta(t)) & \mathcal{D}_{11}(\Theta(t)) & \mathcal{D}_{12}(\Theta(t)) \\
\mathcal{C}_{2}(\Theta(t)) & \mathcal{D}_{21}(\Theta(t)) & \mathcal{D}_{22}(\Theta(t))
\end{array}\right]}\left[\begin{array}{l}
x(t) \\
d(t) \\
u(t)
\end{array}\right],
$$

where $\Theta(t) \in \Theta, \dot{x}, x \in \mathbb{R}^{n}, d \in \mathbb{R}^{n_{d}}$ is the disturbance, $e \in$ $\mathbb{R}^{n_{e}}$ is the controlled output, $u \in \mathbb{R}^{n_{u}}$ is the control input and $y \in \mathbb{R}^{n_{y}}$ is the measurement for control. The underbraced state space matrices of (6) depend on the parameter $\theta$ in a linear fractional way as follows:

$$
\left[\begin{array}{ccc}
A & B_{1} & B_{2} \\
C_{1} & D_{11} & D_{12} \\
C_{2} & D_{21} & D_{22}
\end{array}\right]+\left[\begin{array}{c}
B_{0} \\
D_{10} \\
D_{20}
\end{array}\right] \Theta(t)\left(I-D_{00} \Theta(t)\right)^{-1}\left[\begin{array}{lll}
C_{0} & D_{01} & D_{02}
\end{array}\right] .
$$


It is assumed that the LFT representation is well-posed; that is, $\left(I-D_{00} \Theta(t)\right)$ is invertible for any allowable parameter values. It is also assumed that the triple $\left(\mathcal{A}_{1} \mathscr{B}_{2}, \mathcal{C}_{2}\right)$ is parameter-dependent stabilizable and detectable for all $\theta \in$ $\mathcal{F}_{\mathcal{P}}^{\nu}$. This guarantees the existence of a stabilizing output feedback LPV controller. The class of LPV controllers we are interested in is of the form

$$
\left[\begin{array}{c}
\dot{x}_{k}(t) \\
u(t)
\end{array}\right]=\left[\begin{array}{ll}
\mathcal{A}_{k}(\Theta(t), \dot{\Theta}(t)) & \mathcal{B}_{k}(\Theta(t), \dot{\Theta}(t)) \\
\mathcal{C}_{k}(\Theta(t), \dot{\Theta}(t)) & D_{k}(\Theta(t), \dot{\Theta}(t))
\end{array}\right]\left[\begin{array}{c}
x_{k}(t) \\
y(t)
\end{array}\right],
$$

where $x_{k} \in \mathbb{R}^{n_{k}}$. The dimension of controller state $n_{k}$ is yet to be determined.

The synthesis method used in this paper is based upon the results in [6] for LPV systems and [8] for LTI $\mathscr{H}_{\infty}$ synthesis with pole clustering. The following definition is taken from [8].

Definition 1 (LMI-region). A subset $\mathscr{D}$ of the complex plane is called an LMI region if there exist a symmetric matrix $\alpha=$ $\left[\alpha_{k l}\right] \in \mathbb{R}^{m \times m}$ and a matrix $\beta=\left[\beta_{k l}\right] \in \mathbb{R}^{m \times m}$ such that $\mathscr{D}=\left\{z \in \mathbb{C}: f_{\mathscr{D}}(z)<0\right\}$ with

$$
f_{\mathscr{D}}(z)=\alpha+z \beta+\bar{z} \beta^{T}=\left[\alpha_{k l}+\beta_{k l} z+\beta_{l k} \bar{z}\right]_{1 \leq k, l \leq m} .
$$

These regions make up a dense subset in the set of regions of the complex plane, symmetric with respect to the real axis. This makes them appealing for specifying pole placement design objectives.

Theorem 1 (LPV basic characterization with pole placement constraints). Let $r_{\S}=\left(r_{1}^{\&}, \ldots, r_{s}^{\S}\right)$ and $r_{\mathcal{R}}=\left(r_{1}^{\mathcal{R}}, \ldots, r_{s}^{\mathcal{R}}\right) \in$ $\mathbb{N}^{s}$ define $n_{s}, n_{\mathcal{R}}, \boldsymbol{\Theta}_{\&}, \dot{\boldsymbol{\Theta}}_{\&}$ and $\boldsymbol{\Theta}_{\mathcal{R}}, \dot{\boldsymbol{\Theta}}_{\mathcal{R}}$ as in the beginning of this section. Let $\delta\left(\Theta_{\S}\right)=\mathcal{T}_{\S}\left(\Theta_{\S}\right)^{T} Q \mathcal{T}_{\S}\left(\Theta_{\S}\right)$ and $\mathcal{R}\left(\Theta_{\mathcal{R}}\right)=$ $\widetilde{\mathcal{T}}_{\mathcal{R}}\left(\Theta_{\mathcal{R}}\right)^{T} P \mathcal{T}_{\mathcal{R}}\left(\Theta_{\mathcal{R}}\right)$, with

$$
\begin{gathered}
\mathcal{T}_{\S}\left(\Theta_{\S}\right)=\Theta_{\S} \star\left[\begin{array}{l|l}
T_{\delta_{11}} & T_{\delta_{12}} \\
\hline T_{\delta_{21}} & T_{\delta_{22}}
\end{array}\right], \\
\mathcal{T}_{\mathcal{R}}\left(\Theta_{\mathcal{R}}\right)=\Theta_{\mathcal{R}} \star\left[\begin{array}{l|l}
T_{\mathcal{R}_{11}} & T_{\mathcal{R}_{12}} \\
\hline T_{\mathcal{R}_{21}} & T_{\mathcal{R}_{22}}
\end{array}\right],
\end{gathered}
$$

be two symmetric and positive definite matrix functions, where constant matrices $Q$ and $P$ are yet to be found, with $\Theta_{s} \in \Theta_{s}$ and $\Theta_{\mathcal{R}} \in \Theta_{\mathcal{R}}$. Consider the LPV plant governed by (6), with parameter trajectories in $\mathcal{F}_{\mathcal{P}}^{v}$. Suppose that there exist parameter dependent symmetric matrices $\&$ and $\mathcal{R}$ such as (10), a positive real number $\gamma$, and a parameter dependent quadruple of state space data $\widehat{\mathcal{A}}_{k}, \widehat{B}_{k}, \widehat{\mathcal{C}}_{k}$, and $D_{k}$, such that the following LMI constraints

$$
-\left[\begin{array}{cc}
\mathcal{R} & I \\
I & 8
\end{array}\right]<0
$$

$$
\begin{gathered}
{\left[\alpha_{k l}\left[\begin{array}{cc}
\mathcal{R} & I \\
I & 8
\end{array}\right]+\beta_{k l} \Phi+\beta_{l k} \Phi^{T}\right]_{k, l}<0} \\
\Psi=\left[\begin{array}{ll}
\Psi_{11} & \Psi_{21}^{T} \\
\Psi_{21} & \Psi_{22}
\end{array}\right]<0
\end{gathered}
$$

are satisfied for all $\theta \in \mathcal{P}, \dot{\theta} \in \mathcal{V}$, with

$\Phi$

$$
=\left[\begin{array}{cc}
\mathcal{A} \mathcal{R}+\mathscr{B}_{2} \widehat{C}_{k} & \mathcal{A}+\mathscr{B}_{2} \mathscr{D}_{k} \mathcal{C}_{2} \\
\widehat{\mathscr{A}_{k}} & \mathcal{A A}+\widehat{\mathscr{B}}_{k} \mathcal{C}_{2}
\end{array}\right],
$$

$\Psi_{11}$

$=\left[\begin{array}{cc}-\dot{\mathcal{R}}+\mathcal{A} \mathcal{R}+\mathcal{R} \mathcal{A}^{T}+\mathscr{B}_{2} \widehat{\mathcal{C}}_{k}+\left(\mathcal{B}_{2} \widehat{\mathcal{C}}_{k}\right)^{T} & \mathscr{B}_{1}+\mathscr{B}_{2} \mathscr{D}_{k} \mathscr{D}_{21} \\ \left(\mathcal{B}_{1}+\mathscr{B}_{2} \mathscr{D}_{k} \mathscr{D}_{21}\right)^{T} & -\gamma I\end{array}\right]$,

$\Psi_{21}$

$=\left[\begin{array}{cc}\widehat{\mathcal{A}_{k}}+\left(\mathcal{A}+\mathscr{B}_{2} \mathscr{D}_{k} \mathcal{C}_{2}\right)^{T} & \& \mathscr{B}_{1}+\widehat{\mathcal{B}}_{k} \mathscr{D}_{21} \\ \mathcal{C}_{1} \mathcal{R}+\mathscr{D}_{12} \widehat{C}_{k} & D_{11}+\mathscr{D}_{12} \mathscr{D}_{k} \mathscr{D}_{21}\end{array}\right]$,

$\Psi_{22}$

$=\left[\begin{array}{cc}\dot{\delta}+\& \mathcal{A}+\mathcal{A}^{T} \&+\widehat{\mathscr{B}}_{k} \mathcal{C}_{2}+\left(\widehat{\mathscr{B}}_{k} \mathcal{C}_{2}\right)^{T} & \left(\mathcal{C}_{1}+\mathscr{D}_{12} \mathcal{D}_{k} \mathcal{C}_{2}\right)^{T} \\ \mathcal{C}_{1}+\mathscr{D}_{12} \mathscr{D}_{k} \mathcal{C}_{2} & -\gamma I\end{array}\right]$.

Then, there exists a gain-scheduled output-feedback controller as (8) such that one has the following.

(1) Internal stability is enforced.

(2) $\gamma$ is a bound on the $L_{2}$ gain of the closed-loop system given by the interconnection of (6) with (8).

(3) The poles of each closed-loop LTI system, resulting from all constant parameter trajectories in $\mathcal{F}_{\mathcal{P}}^{v}$, are circumscribed to an LMI region prescribed by a characteristic function such as (9).

Proof. See $[8,11]$.

For the optimization problem to be convex, this method seeks a unique closed loop Lyapunov Matrix $\mathcal{X}$ simultaneously valid for $L_{2}$-gain and pole placement conditions. $\mathcal{X}$ can be computed from $\&$ and $\mathcal{R}$. As mentioned in [8], this approach is potentially conservative, but rarely in practice.

2.2. Main Results. Next, to proceed towards the derivation of synthesis conditions, a dependence of the so-called transformed controller matrices $\widehat{\mathscr{A}}_{k}, \widehat{\mathscr{B}}_{k}, \widehat{C}_{k}$, and $\mathscr{D}_{k}$ on the measured parameter vector $(\theta)$ is proposed. Let $r_{a_{k}}=$ $\left(r_{1}^{a_{k}}, \ldots, r_{s}^{a_{k}}\right)$ and $r_{c_{k}}=\left(r_{1}^{c_{k}}, \ldots, r_{s}^{c_{k}}\right) \in \mathbb{N}^{s}$ define the numbers $n_{a_{k}}$ and $n_{c_{k}}$, and the sets $\Theta_{a_{k}}, \dot{\Theta}_{a_{k}}$ and $\Theta_{c_{k}}, \dot{\Theta}_{c_{k}}$ as in 
the beginning of this section. The transformed controller matrices will be given by

$$
\begin{array}{rlrl}
\widehat{\mathcal{A}}_{k} & =\mathcal{T}_{a k} \overline{A_{k}}, & \widehat{\mathcal{B}}_{k} & =\mathcal{T}_{a k} \overline{B_{k}}, \\
\widehat{\mathcal{C}_{k}} & =\mathcal{T}_{c k} \overline{C_{k}}, & \mathcal{D}_{k}=\mathcal{T}_{c k} \overline{D_{k}}
\end{array}
$$

with $\overline{A_{k}}, \overline{B_{k}}, \overline{C_{k}}$, and $\overline{D_{k}}$ being constant matrices and

$$
\begin{aligned}
& \mathcal{T}_{a_{k}}\left(\Theta_{a_{k}}\right)=\Theta_{a_{k}} \star\left[\begin{array}{c|c}
T_{a_{k 11}} & T_{s_{12}} \\
\hline T_{\delta_{21}} & T_{\delta_{22}}
\end{array}\right], \quad \Theta_{a_{k}} \in \Theta_{a_{k}}, \\
& \mathcal{T}_{c_{k}}\left(\Theta_{c_{k}}\right)=\Theta_{c_{k}} \star\left[\begin{array}{c|c}
T_{\mathcal{R}_{11}} & T_{\mathcal{R}_{12}} \\
\hline T_{\mathcal{R}_{21}} & T_{\mathcal{R}_{22}}
\end{array}\right], \quad \Theta_{c_{k}} \in \Theta_{c_{k}} .
\end{aligned}
$$

In order to apply the FBSP on LMIs (11), (12), and (13) in a way resembling theorem 4 in [6], the following lemma is presented.

Lemma 2. Let $\Theta_{\mathcal{R}} \in \boldsymbol{\Theta}_{\mathcal{R}}, \Theta_{s} \in \Theta_{s}, \dot{\Theta}_{s} \in \dot{\Theta}_{s}, \dot{\Theta}_{\mathcal{R}} \in \dot{\Theta}_{\mathcal{R}}$, $\Theta_{a_{k}} \in \Theta_{a_{k}}, \Theta_{c_{k}} \in \Theta_{c_{k}}$, and $\Theta \in \Theta$. LMIs (11), (12) and (13) can be rewritten as

$$
g^{T}(-M) g<0, \quad g_{p p}^{T} M_{p p} g_{p p}<0, \quad g_{\infty}^{T} M_{\infty} g_{\infty}<0 .
$$

The $g_{,}, g_{p p}$, and $g_{\infty}$ are LFTs depending on the open loop data (6) and on the $\mathcal{T}_{f}, \mathcal{T}_{\mathcal{R}}, \mathcal{T}_{a_{k}}$, and $\mathcal{T}_{c_{k}}$ functions. They can be expressed as

$$
\begin{gathered}
g=\widehat{\Theta} \star\left[\begin{array}{l|l}
G_{11} & G_{12} \\
\hline G_{21} & G_{22}
\end{array}\right], \\
g_{p p}=\hat{\Theta}_{p p} \star\left[\begin{array}{c|c}
G_{p p_{11}} & G_{p p_{12}} \\
\hline G_{p p_{21}} & G_{p p_{22}}
\end{array}\right], \\
g_{\infty}=\hat{\Theta}_{\infty} \star\left[\begin{array}{c|c}
G_{\infty_{11}} & G_{\infty_{12}} \\
\hline G_{\infty_{21}} & G_{\infty_{22}}
\end{array}\right]
\end{gathered}
$$

with $\widehat{\Theta}=\operatorname{diag}\left(\Theta_{\mathcal{R}}, \Theta_{s}\right), \widehat{\Theta}_{p p}=\operatorname{diag}\left(\Theta_{s}, \Theta_{\mathcal{R}}, \Theta_{a_{k}}, \Theta_{c_{k}}, \Theta\right)$ and $\hat{\Theta}_{\infty}=\operatorname{diag}\left(\dot{\Theta}_{s}, \dot{\Theta}_{\mathcal{R}}, \Theta_{s}, \Theta_{\mathcal{R}}, \Theta_{a_{k}}, \Theta_{c_{k}}, \Theta\right)$. On the other hand, the $M, M_{p p}$, and $M_{\infty}$ matrices depend on the $\alpha$ and $\beta m \times m$ matrices that specify a design LMI region, as in Definition 1, and on

(1) a pair of symmetric positive definite $P$ and $Q$ matrices of the $\&$ and $\mathcal{R}$ matrix functions,

(2) a quadruple $\overline{A_{k}}, \overline{B_{k}}, \overline{C_{k}}$, and $D_{k}$ of controller state space data,

(3) a real positive performance index $\gamma$,

where the enumerated objects are to be determined in the optimization process.

Proof. See [12] for the definition of matrices $M, M_{p p}, M_{\infty}$, $g, g_{p p}$, and $g_{\infty}$ and for the proof, which can be obtained through tedious but straightforward matrix calculations based upon the results in $[6,8]$.
Theorem 2 (LPV control with pole placement constraints and FBMs). The inequalities of (17) are satisfied, if and only if there exist symmetric positive definite real matrices $P$ and $Q$, a performance index $\gamma$, a quadruple of controller matrices $\overline{A_{k}}$, $\overline{B_{k}}, \overline{C_{k}}$, and $\overline{D_{k}}$, and symmetric full block multipliers $\Pi, \Pi_{p p}$ and $\Pi_{\infty}$ such that the following LMIs

$$
\begin{gathered}
{[*]^{T} \operatorname{diag}\{\Pi, M\} \hat{G}<0,} \\
{[*]^{T} \operatorname{diag}\left\{\Pi_{p p}, M_{p p}\right\} \hat{G}_{p p}<0,} \\
{[*]^{T} \operatorname{diag}\left\{\Pi_{\infty}, M_{\infty}\right\} \hat{G}_{\infty}<0,} \\
\hat{G}=\left[\begin{array}{cc}
G_{11} & G_{12} \\
I & 0 \\
G_{21} & G_{22}
\end{array}\right], \\
\hat{G}_{p p}=\left[\begin{array}{cc}
G_{p p_{11}} & G_{p p_{12}} \\
I & 0 \\
\frac{I}{G_{p p_{21}}} & G_{p p_{22}}
\end{array}\right], \\
\hat{G}_{\infty}=\left[\begin{array}{cc}
G_{\infty_{11}} & G_{\infty_{12}} \\
I & 0 \\
\frac{I}{G_{\infty_{21}}} & G_{\infty_{22}}
\end{array}\right]
\end{gathered}
$$

are satisfied, and for all $\theta \in \mathcal{P}$ and $\dot{\theta} \in \mathcal{V}$, the following conditions

$$
\begin{gathered}
{\left[\begin{array}{c}
I \\
\widehat{\Theta}_{P}
\end{array}\right]^{T} \Pi_{P}\left[\begin{array}{c}
I \\
\widehat{\Theta}_{P}
\end{array}\right] \geq 0,} \\
{\left[\begin{array}{c}
I \\
\hat{\Theta}_{Q}
\end{array}\right]^{T} \Pi_{Q}\left[\begin{array}{c}
I \\
\hat{\Theta}_{Q}
\end{array}\right] \geq 0,} \\
{\left[\begin{array}{l}
I \\
\widehat{\Theta}
\end{array}\right]^{T} \Pi\left[\begin{array}{c}
I \\
\widehat{\Theta}
\end{array}\right] \geq 0}
\end{gathered}
$$

are met.

Proof. The application of Lemma 1 to inequalities (17) produces the desired result.

The computation of the controller's state space matrices is carried out following the algorithm prescribed in [7]. All remarks made in that paper, concerning the use of PDLFs, which aim towards obtaining controllers whose state space matrices do not depend on the parameter rate of variation, are applicable here as well (see [7, Table I]).

As observed in [6], in practice, the considerations of Remark 1 can lead to significantly reducing the number of decision variables of the problem. Degradation of the computed performance bound $\gamma$ is also a possibility. 


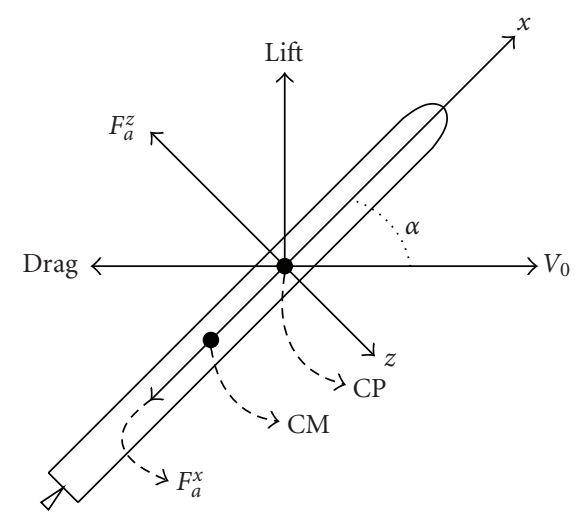

(a)

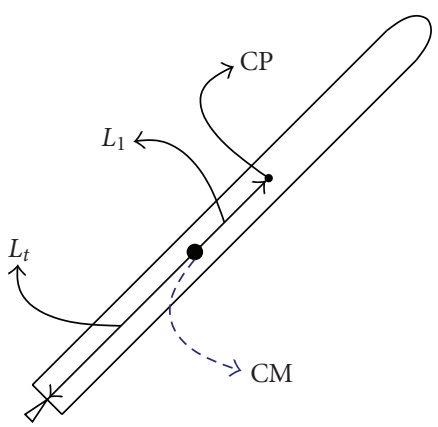

(b)

FIgURE 1: Vehicle diagram.

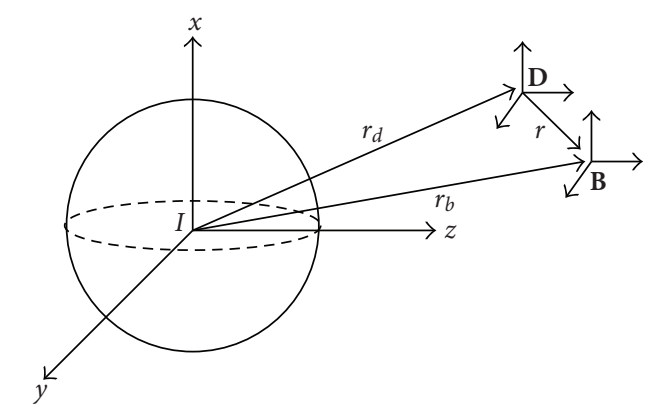

Figure 2: Position of frame B with respect to frame D.

\section{Application Example}

3.1. Nonlinear Model. The example we consider is a sounding rocket (see Figure 1) which should follow a prescribed trajectory. A dynamic model is presented (see Figure 2), describing the position, velocity, orientation, and angular velocity errors of the actual vehicle (frame B) with respect to the prescribed trajectory (frame $\mathbf{D}$ ). The differential gravity force is neglected in this error model for practical reasons.

A simple model of aerodynamic drag and lift forces taken from [13] is included. According to this model, these forces depend on the dynamic pressure, the angles of attack $\alpha$, and sideslip $\beta$ (see [14]). The moments resulting from the aerodynamic forces are computed under the assumption that the center of pressure $(\mathrm{CP})$ is located above the CM. This renders unstable aerodynamics posing a challenge on the control system (see Figure 1). $F_{a}^{x}, F_{a}^{y}$, and $F_{a}^{z}$ denote the aerodynamic forces acting on the $\mathrm{CP}$ in frame $\mathbf{B}$.

The actuator considered for the rocket is a nozzle gimbal which allows small rotations around the $z$-and $y$-axes. A couple of gas jets are placed to generate torques around the $x$-axis. Hence, the actual control inputs of the plant will be the thrust, the rotation angles of the gimbal, and the torque exerted by the jets. For small rotations of the gimbal, a change of variables is carried out, and as a consequence, the control inputs of the plant model used in the controller design will be denoted by $F_{t h}^{x}, F_{t h}^{y}, F_{t h}^{z}$, and $m_{x}$ assuming that the forces are applied at the tail (hence providing torques in the $y$ and $z$ axes). The following terms will be used to denote the actuator and aerodynamic forces and moments in the state space equations:

$$
\begin{aligned}
& F=\left[\begin{array}{lll}
\left(F_{t h}^{x}+F_{a}^{x}\right) & \left(F_{t h}^{y}+F_{a}^{y}\right) & \left(F_{t h}^{z}+F_{a}^{z}\right)
\end{array}\right]^{T}, \\
& M=\left[\begin{array}{ll}
m_{x}-\left(\ell F_{t h}^{z}+\ell_{1} F_{a}^{z}\right) & \left(\ell F_{t h}^{y}+\ell_{1} F_{a}^{y}\right)
\end{array}\right]^{T} .
\end{aligned}
$$

$F_{d}$ and $M_{d}$ denote the counterparts of $F$ and $M$ for the desired trajectory. As mass variation concerns, it is assumed that the thrust force acting on the vehicle comes from fuel exhaustion and that the inertia matrix varies uniformly with it, that is, the CM does not change. $J=\operatorname{diag}\left[\begin{array}{lll}J_{x} & J_{t} & J_{t}\end{array}\right]$ denotes the diagonal varying inertia matrix. The variables for the vehicle dynamics will be $r, v,\left[q_{0}, q\right]$, and $\omega$ which, respectively, give the position, velocity, orientation and angular velocity of frame $\mathbf{B}$ relative to frame $\mathbf{D}$. Under these assumptions, the error dynamics of the rocket are

$$
\begin{gathered}
\dot{r}=v, \quad \dot{v}=\frac{1}{m}\left({ }^{d} C_{b} F-F_{d}\right), \\
\dot{q}=\frac{1}{2}\left(q_{0} \omega+q \times \omega\right), \quad \dot{\omega}=J^{-1}\left({ }^{d} C_{b} M-M_{d}\right), \\
\dot{q}_{0}=-\frac{1}{2} q \cdot \omega
\end{gathered}
$$

with ${ }^{d} C_{b}$ denoting the rotation matrix from frame $\mathbf{B}$ to $\mathbf{D}$.

3.2. Linearization: LPV Model. Linearization of (22) will be carried out, in order to obtain an LPV model of the plant, under a few extra assumptions related to nominal control and aerodynamic forces. During ascent, the vehicle should nominally follow a zero angle of attack trajectory, hence only withstanding drag. It can be shown that the nominal actuator forces acting on the $y$-and $z$-axes of the vehicle are negligible as compared to the actual forces that compensate for disturbances when the actual angle of attack deviates from zero. It can also be accepted that $m_{x}$ is nominally zero. Under these assumptions $M_{d}=0$, and 
$F_{d}=\left[\begin{array}{lll}\left(f_{N}-D\right) & 0 & 0\end{array}\right]^{T}$ where $f_{N}$ is the nominal thrust and $D$ is the drag force. Let $u \triangleq\left[\begin{array}{llll}F_{t h}^{x} & F_{t h}^{y} & F_{t h}^{z} & m_{x}\end{array}\right]^{T}$ and $u_{d} \triangleq\left[\begin{array}{llll}f_{N} & 0 & 0 & 0\end{array}\right]^{T}$. Let $r_{i}, v_{i}, q_{i}$, and $\omega_{i}(i=1,2,3)$ be the components of the corresponding vectors, and the state variable is $x \triangleq\left[\begin{array}{llll}x_{1}^{T} & x_{2}^{T} & x_{3}^{T} & x_{4}^{T}\end{array}\right]^{T}$ with $x_{1}=\left[\begin{array}{ll}r_{1} & v_{1}\end{array}\right]^{T}, x_{2}=$

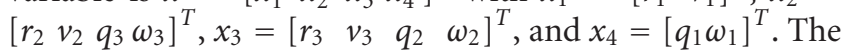
differential control is $\delta_{u}=u-u_{d}$. With these definitions in mind we seek to linearize (22) which can be rewritten as

$$
\dot{x}=f\left(x, \delta_{u}, \theta\right)=A(\theta) x+B(\theta) \delta_{u}+o\left(x, \delta_{u}, \theta\right),
$$

where $o\left(x, \delta_{u}, \theta\right)$ represents the higher-order terms of the series expansion of $f$ around $(0,0, \theta) . A(\theta)$ and $B(\theta)$ are the following Jacobians:

$$
\left.\left.A(\theta) \triangleq \frac{\partial f\left(x, \delta_{u}, \theta\right)}{\partial x}\right|_{(0,0, \theta)} B(\theta) \triangleq \frac{\partial f\left(x, \delta_{u}, \theta\right)}{\partial \delta_{u}}\right|_{(0,0, \theta)} .
$$

The LPV parameter $\theta$ is a function of mass, dynamic pressure, and the state variables of the actual vehicle. Mass variations can be estimated with a model of fuel consumption, and dynamic pressure can be estimated from inertial position and velocity measurements as well as from a model of the atmosphere. Hence, $\theta$ can be known in real time as if it were measured.

An evaluation of the Jacobian matrices of (24) through a symbolic manipulation software package shows that $A(\theta)$ and $B(\theta)$ are affine in the parameters, as follows:

$$
\theta_{1} \triangleq \frac{2 Q_{0}}{m S}, \quad \theta_{2} \triangleq \frac{1}{m}
$$

It also shows that the system can be decomposed in four decoupled systems corresponding to the $x_{i}$ parts of the state variable $x(i=1,2,3,4)$. As a consequence, the control problem can be split in four $\gamma$-performance problems with pole placement constraints with each subsystem having a state space representation as follows $(i=1,2,3,4)$ :

$$
\dot{x}_{i}=A_{i}(\theta) x+B_{i}(\theta) \delta_{u_{i}}, \quad \theta=\left[\begin{array}{ll}
\theta_{1} & \theta_{2}
\end{array}\right]^{T} .
$$

$\theta$ evolves in the parameter variation set which is a square in $\mathbb{R}^{2}$. Mass and dynamic pressure variations throughout the nominal trajectory determine the bounds for $\theta_{1}$ and $\theta_{2}$.

The aerodynamic forces are included in the error dynamics in a first-order approximation for zero angle of attack nominal trajectories as follows:

$$
F_{a}^{z}=\left(\left.\frac{\partial F_{a}^{z}}{\partial q_{y}}\right|_{(0,0, \theta)}\right) q_{y}, \quad F_{a}^{y}=\left(\left.\frac{\partial F_{a}^{y}}{\partial q_{z}}\right|_{(0,0, \theta)}\right) q_{z} .
$$

These terms end up inside the $A_{i}$ matrices of the LPV plants for $i=2,3$ with the derivatives depending on $\theta_{1}$. Since drag has a maximum for the nominal trajectory, its variations are neglected in the first-order approximation. Hence, aerodynamics only affect subsystems 2 and 3.
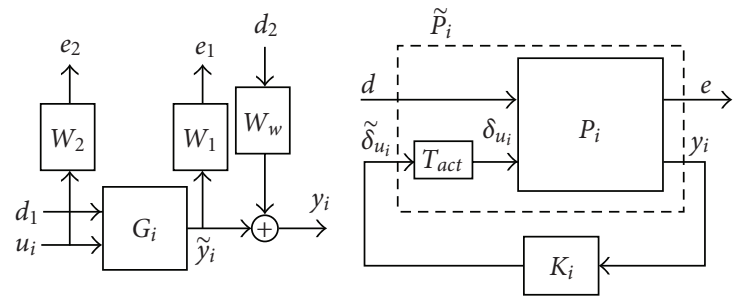

FIGURE 3: Block interconnection making up the augmented plant (left) and Inclusion of low-pass transfer function model of actuator dynamics (right).

3.3. Controller Synthesis. As a consequence of decoupling, the control problem is reduced to synthesizing one controller per subsystem. This simplifies the statement since systems $G_{1}$ and $G_{4}$ depend only on parameter $\theta_{2}$.

For each subsystem an augmented plant as the one in (6) must be specified. In this problem, for all subsystems, the disturbance signal is split into a part which represents disturbance forces or moments, and a part which represents measurement noise. The performance signal is divided in two parts as well. One of them involves the state variables and it represents the design's commitment with disturbance rejection at the output. The other one involves the control force and is included in order to achieve disturbance rejection with reasonable control action.

For each subsystem the augmented plant is the result of the block interconnection of Figure 3. The state space representation of each $G_{i}$ block is as follows:

$$
\begin{gathered}
\dot{x}_{i}=\mathcal{A}_{i}(\theta) x_{i}+\mathcal{B}_{d_{i}}\left(\theta_{2}\right) d_{1}+\mathcal{B}_{i}\left(\theta_{2}\right) \delta_{u_{i}}, \\
\tilde{y}_{i}=x_{i} .
\end{gathered}
$$

For the sake of clarity as notation regards, note that the $\mathscr{B}_{i}$ matrices of the systems described by (28) play the role of matrix $\mathscr{B}_{2}$ in the system described by (6). For the final augmented plants $\left(\widetilde{P}_{i}\right.$, Figure 3$)$ the lowpass transfer function $T_{\text {act }}(s)=1 /\left(\left(s / \omega_{a}\right)+1\right)$, with $\omega_{a}=2 \pi \cdot 10 \mathrm{rad} / \mathrm{sec}$, is included in order to model actuator dynamics. As the $B_{d_{i}}$ matrix concerns, it is assumed for all systems that $B_{d_{i}}=\xi_{i} B_{i}$ with $\xi_{i}$ being a design parameter $\left(1<\xi_{i}<10\right)$. The $\xi_{i}$ gives the ratio between the disturbance and control forces or torques.

System $G_{1}$ involves longitudinal dynamics. The matrices that make up the plant are as follows:

$$
\begin{aligned}
A_{1} & =\left[\begin{array}{ll}
0 & 1 \\
0 & 0
\end{array}\right], \\
W_{w} & =\left[\begin{array}{ll}
50 & 0 \\
0 & .2
\end{array}\right], \\
B_{1} & =\left[\begin{array}{l}
0 \\
\theta_{2}
\end{array}\right]
\end{aligned}
$$

with $W_{1}=W_{w}^{-1}, k_{u_{1}}=10^{-2}$ and $\xi_{1}=10$. 
Systems $G_{2}$ and $G_{3}$ involve lateral dynamics, with their matrices being

$$
\begin{gathered}
A_{2}=\left[\begin{array}{cccr}
0 & 1 & 0 & 0 \\
0 & 0 & -D_{\alpha} \theta_{1}+2 f_{N} \theta_{2} & 0 \\
0 & 0 & 0 & \frac{1}{2} \\
0 & 0 & 0 & 0
\end{array}\right], \\
A_{3}=\left[\begin{array}{cccc}
0 & 1 & 0 & 0 \\
0 & 0 & D_{\alpha} \theta_{1}-2 f_{N} \theta_{2} & 0 \\
0 & 0 & 0 & \frac{1}{2} \\
0 & 0 & 0 & 0
\end{array}\right], \\
B_{2}=\left[\begin{array}{cccc}
0 & 0 \\
\theta_{2} & \theta_{2} \\
0 & B_{3}= \\
-\frac{m_{0}}{I_{t_{0}}} \theta_{2}
\end{array}\right] \\
W_{w}
\end{gathered}
$$

with $W_{1}=W_{w}^{-1}, k_{u_{2}}=k_{u_{3}}=10^{-2}$, and $\xi_{2}=\xi_{3}=10$.

System $G_{4}$ involves roll dynamics. The matrices that make up the plant are as follows:

$$
\begin{gathered}
A_{4}=\left[\begin{array}{ll}
0 & \frac{1}{2} \\
0 & 0
\end{array}\right], \\
W_{w}=\left[\begin{array}{cc}
\frac{\theta}{180} & 0 \\
0 & .1 \frac{\theta}{180}
\end{array}\right], \\
B_{4}=\left[\begin{array}{c}
0 \\
\frac{m_{0}}{I_{x 0}} \theta_{2}
\end{array}\right]
\end{gathered}
$$

with $W_{1}=W_{w}^{-1}, k_{u_{4}}=10^{-2}$, and $\xi_{4}=10$.

3.4. Synthesis Results. The synthesis procedure was carried out on all subsystems. The pole placement region used was $\mathscr{D}=\{z \in \mathbb{C},-2 \pi \cdot 8 \mathrm{rad} / \mathrm{sec}<\mathfrak{R}(z)\}$, which takes into account practical aspects such as ease of implementation and simulation. The vehicle's details can be seen in Table 1 . In this example, focus is made on demonstrating the validity of the presented synthesis method. As a consequence, in
TABLE 1: Vehicle's details.

\begin{tabular}{lcc}
\hline Name & Symbol & Value \\
\hline Initial/final Mass & $m_{0} / m_{f}$ & $500 / 250 \mathrm{~kg}$ \\
Initial/final Inertia & $J_{x_{0}} / J_{x_{f}}$ & $60 / 30 \mathrm{~kg} \mathrm{~m}^{2}$ \\
Initial/final Inertia & $J_{t_{0}} / J_{t_{f}}$ & $200 / 100 \mathrm{~kg} \mathrm{~m}^{2}$ \\
& $D_{\alpha}=\frac{\partial \bar{F}_{a}^{z}}{\partial q_{2}}$ & 0.1963 \\
Aerodynamic Derivative & $f_{n}$ & $20 \times 10^{3} \mathrm{~N}$ \\
Nominal thrust & $\ell$ & $2 \mathrm{~m}$ \\
Distance from CM to nozzle & $\ell_{1}$ & $1 \mathrm{~m}$ \\
Distance from CM to CP & $Q_{0_{\max }}$ & $3 \times 10^{5} \mathrm{~N} / \mathrm{m}^{2}$ \\
Maximum Dynamic Pressure
\end{tabular}

order to compare the results obtained here with respect to previous work [10], SQLFs were employed on each subsystem (i.e., $\&$ and $\mathcal{R}$ constant). Another design decision that was taken concerns the choice of the $\mathcal{T}_{a_{k}}$ and $\mathcal{T}_{c_{k}}$ functions which prescribe the way controller matrices dependend on the parameter. For the example these functions were picked as

$$
\widetilde{T}_{a_{k}}=\left[\begin{array}{lll}
I_{n} & \theta_{1} I_{n} & \theta_{2} I_{n}
\end{array}\right], \quad \mathcal{T}_{c_{k}}=\left[\begin{array}{lll}
I_{n_{u}} & \theta_{1} I_{n_{u}} & \theta_{2} I_{n_{u}}
\end{array}\right] .
$$

This choice was made, in order to have the same kind of affine parameter dependence of the original plant in the controller. Once the controllers for each subsystem were synthesized, they were appended to make up the complete LPV controller.

A heuristic approach to address the "fast poles" problem, while using algorithms as the one presented in [6], is to bound the trace of the $\mathcal{R}$ and $\&$ matrices. A bound in the range of the decision variables could be imposed alternatively. The method is occasionally successful but offers no guarantee. In this particular application, this heuristic was useless in order to prevent one single "fast pole" in the controller, per subsystem. Another heuristic was tried, consisting in the residualization of the "fast pole" in the LTI part of the controller. Nevertheless, this approach showed poor closed loop transient behavior in non-realtime simulations. As a consequence, it was dropped. On the other hand, the controller synthesized using the proposed method showed better time responses with lower overshoot. The performance $\gamma$ s with the standard algorithm [6] were in the order of the ones obtained with the proposed one. The overall conclusion is that the method proposed enhances the capabilities of the standard LPV technique for real-world applications.

A considerable increase in the number of decision variables can take place. For this application, with the pole placement region being a half plane, the number of decision variables of the optimization problem for each lateral controller goes from 73 with the algorithm of [6] to 1363 in this case. A more careful and potentially conservative restriction of the number of multiplier variables, in line with Remark 1, could reduce this number. This was not done in this case, given that the computation time using standard PC 


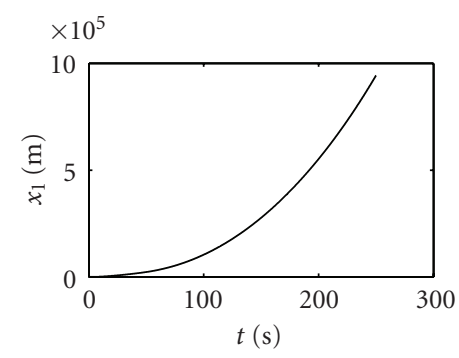

(a)

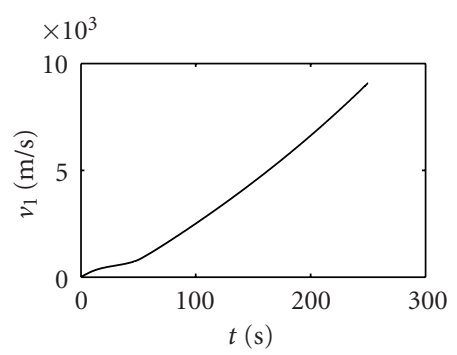

(d)

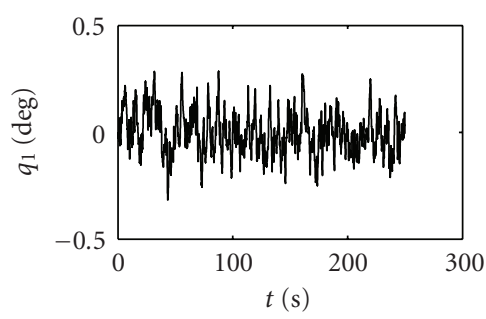

(g)

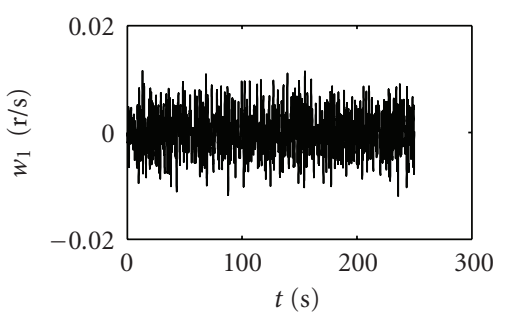

(j)

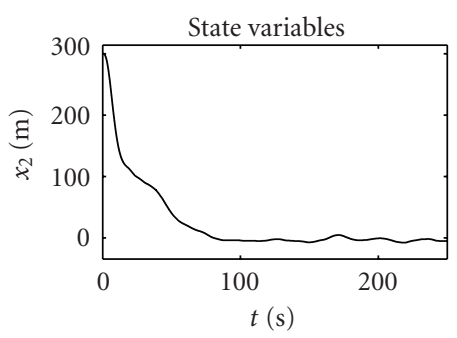

(b)

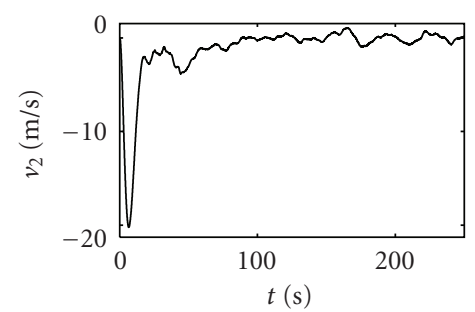

(e)

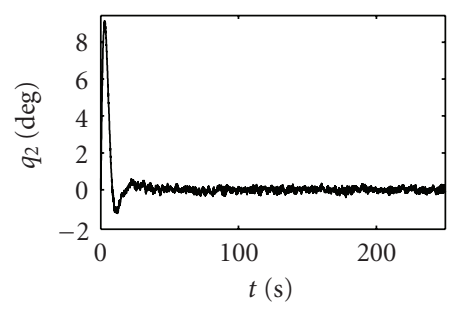

(h)

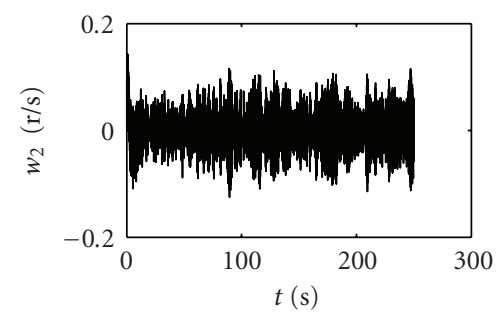

(k)

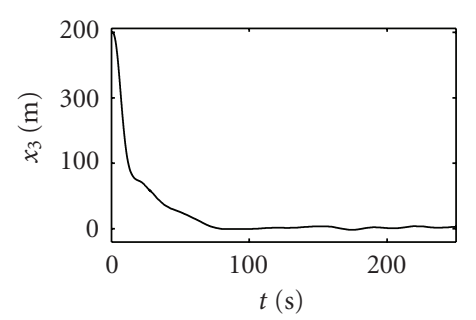

(c)

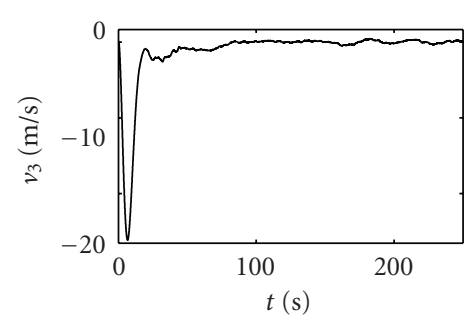

(f)

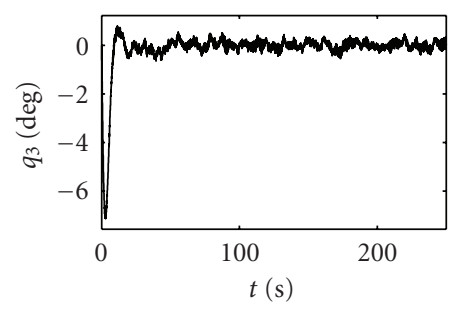

(i)

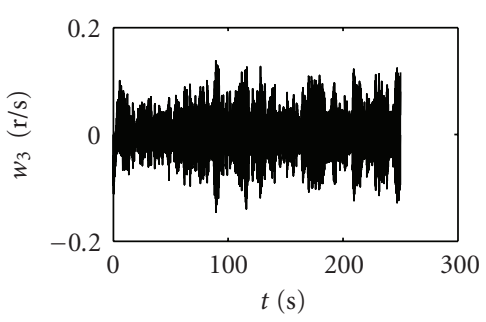

(l)

Figure 4: Response to initial conditions. Error State Variables versus time (sec). (a), (b), and (c): position components (m). (d), (e), and (f): velocity components $(\mathrm{m} / \mathrm{sec})$ ). (g), (h), and (i): quaternion components (deg). (j), (k), and (l): angular velocity components (rad/sec).

hardware was acceptable and considering that the number of decision variables does not influence the implementation of the controller.

With respect to previous work [10], the improvement in the performance bounds is a remarkable result $(\gamma=$ 1.89 versus 162 for subsystem $1, \gamma=30.46$ versus 96 for subsystems 2 and 3 , and $\gamma=1.29$ versus 169 for subsystem 4 ). Moreover, an improvement in the reduction of the sampling frequency from 125 to $45.45 \mathrm{~Hz}$ due to the feasible relocation of closed loop poles was achieved.

3.5. HIL Simulations. To stress the fact that the method is not only valid but also applicable, real-time numerical simulations were carried out. The control law was implemented in a computer based upon an Atmel TSC695E SPARC7 class microprocessor operating at $20 \mathrm{MHz}$. This 32-bit microcontroller has been available in commercial space systems for more than a decade, setting a de facto standard. The setup for simulation is fairly simple, consisting of an Atmel VAB695E Evaluation Kit, with an add-on board containing an Atmel AT7908E CANbus (ISO 118981) controller, connecting this computer with a standard PC featuring a Peak-PCAN pci CANbus interface board. The use of CANbus in aerospace applications is fairly standard [15]. The simulation of the vehicle's dynamics is carried out on the PC using GNU Scientific Library [16], for the real-time simulation of the vehicle's dynamics. The 


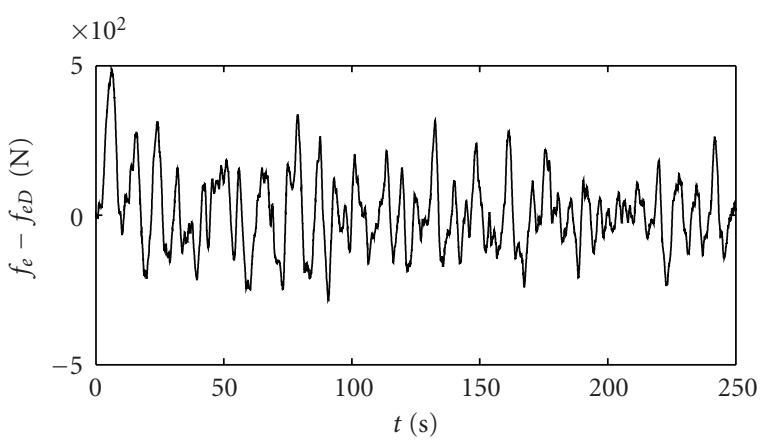

(a)

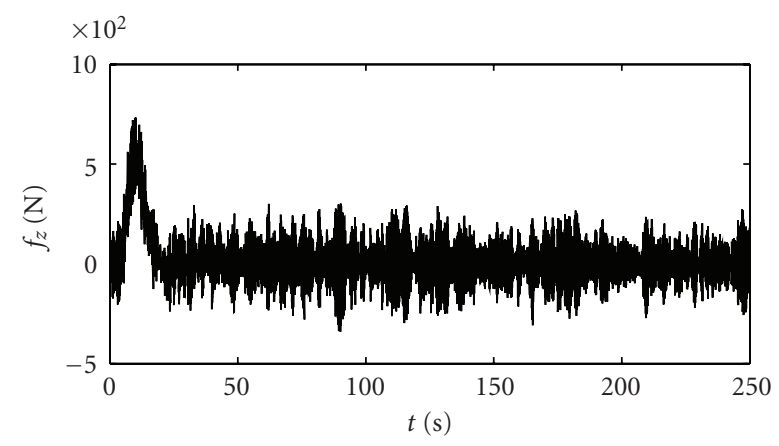

(c)

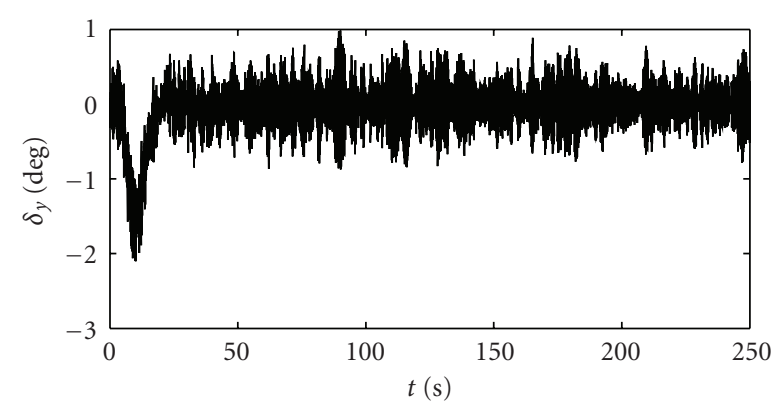

(e)

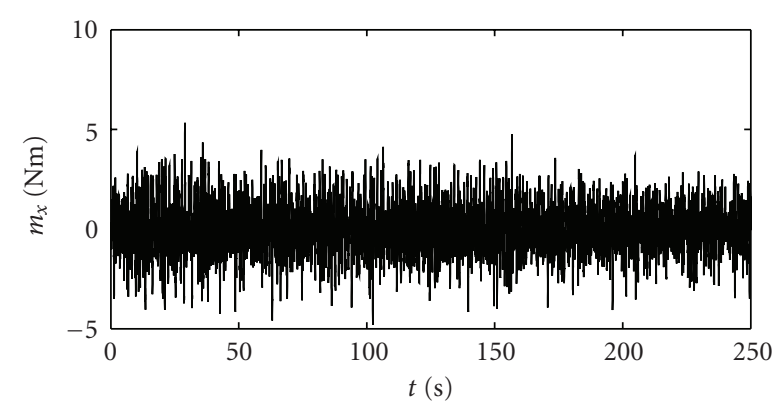

(b)

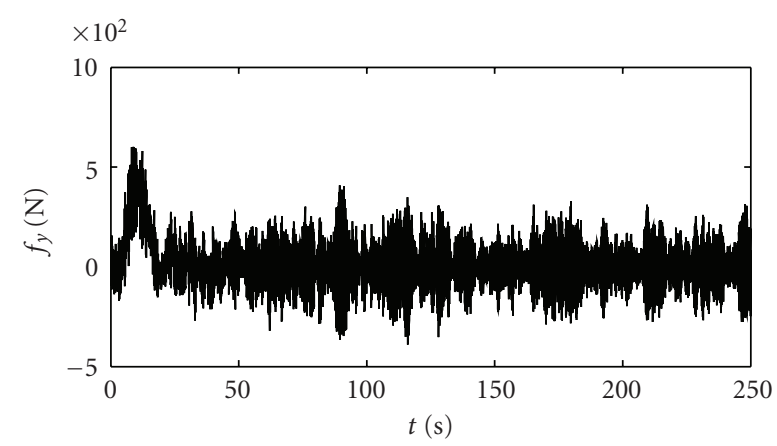

(d)

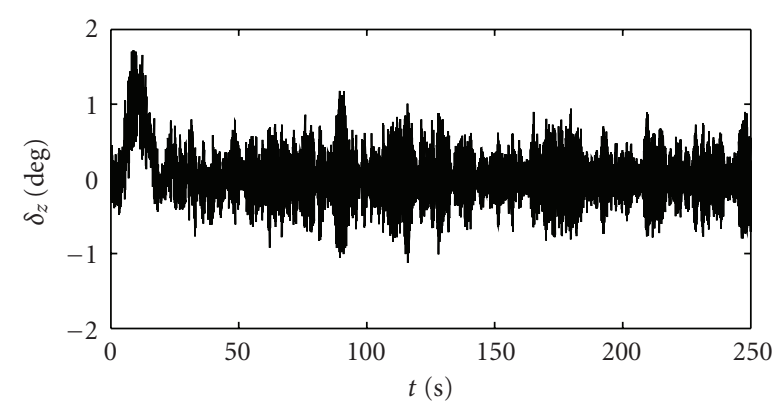

(f)

Figure 5: Response to initial conditions. Column 1: error Control Forces $F_{x}(\mathrm{~N}), F_{y}(\mathrm{~N}), F_{z}(\mathrm{~N})$, and $m_{x}(\mathrm{Nm})$ versus time (sec). Column 2: error commands $f_{e}(\mathrm{~N}), \delta_{y}(\mathrm{deg}), \delta_{z}(\mathrm{deg})$ and $m_{x}(\mathrm{Nm})$ versus time as well.

PC features Xenomai Linux [17] as Real-Time Operating System. GNU Scientific Library is employed in the VAB695E software as well, in single precision floating point mode, for the implementation of the LPV control law. RTEMS real-time operating system [18] features the VAB695E board.

To evaluate the response of the system to disturbance signals the $L_{2}$-gain criterion accounts for, the PC simulates sensor measurements corrupted by colored, weighted, pseudorandom noise. An input disturbance is introduced as well consisting of colored, weighted, pseudorandom lateral forces. These corrupted simulated measurements are transmitted upon request from the control computer. The VAB695E computes control inputs in real time, transmitting commands to actuators simulated in the PC. This renders simulated real time closed loop operation.
The simulations carried out show responses with the initial conditions deviated from the nominal ones as follows:

$$
x(0)=\left[\begin{array}{lllll}
r(0)^{T} & v(0)^{T} & q_{0}(0) & q(0)^{T} & \omega(0)^{T}
\end{array}\right]^{T}
$$

with

$$
\begin{gathered}
r(0)=\left[\begin{array}{lll}
6378000 & 300 & 300
\end{array}\right]^{T}, \quad v(0)=\left[\begin{array}{lll}
0 & .1 & .1
\end{array}\right]^{T}, \\
q(0)=\left[\begin{array}{lll}
0 & .0316 & 0
\end{array}\right]^{T}, \quad \omega(0)=\left[\begin{array}{lll}
0 & 0 & 0
\end{array}\right]^{T}, \quad q_{0}(0)=.995 .
\end{gathered}
$$

All quantities in bold face should nominally be zero, except for $q_{0}$ which would be nominally one. The prescribed trajectory is a vertical ascent from the north pole. Disturbance of the initial conditions is useful in order to evaluate transient behavior. 
In Figure 4 the time history of the state variables can be seen. Figure 5 shows the control error forces and commands. The gimbal's rotations $\left(\delta_{y}\right.$ and $\left.\delta_{z}\right)$ are simulated with a saturation at $5^{\circ}$. Note nevertheless that they remain unsaturated practically throughout the whole simulation. The discrete time implementation of the controller, which is synthesized in a continuous time framework, is carried out through a zero-order hold transformation on the LTI part of the LFT controller (see [19]). The sampling frequency picked for this was $45.45 \mathrm{~Hz}$ (22-millisecond sampling time).

\section{Conclusions}

In this work, the use of FBMs was extended to LPV synthesis with regional pole placement constraints. The usefulness of the method was tested on an application example with HIL simulations. The design of an LPV controller for a 6 DOF vehicle with pole placement constraints shows an adequate response without degrading the $\gamma$ performance index. The system's LPV "poles" were satisfactorily placed acceptably increasing the computational cost of design.

\section{Acknowledgments}

This research was partially supported by the Universidad Nacional de Quilmes, Argentina, through Grant PUNQ 0530/07. The setup for simulation was assembled at the laboratories of CONAE, the Argentine Space Agency. The second author has been supported by CONICET and a PRH Grant from the Ministry of Science and Technology of Argentina. The authors wish to acknowledge the contribution of Dr. Ke Dong to this work, who gently sent the source code of the software developed for the example in [6], for us to see.

\section{References}

[1] C. W. Scherer, Advances in Linear Matrix Inequality Methods in Control, chapter 10, SIAM, Philadelphia, Pa, USA, 1999.

[2] C. W. Scherer, "LPV control and full block multipliers," Automatica, vol. 37, no. 3, pp. 361-375, 2001.

[3] F. Wu, "An unified framework for LPV system analysis and control synthesis," in Proceedings of the IEEE Conference on Decision and Control, vol. 5, pp. 4578-4583, 2000.

[4] F. Wu, "A generalized LPV system analysis and control synthesis framework," International Journal of Control, vol. 74, no. 7, pp. 745-759, 2001.

[5] F. Wu, X. H. Yang, A. Packard, and G. Becker, "Induced L2-norm control for LPV systems with bounded parameter variation rates," International Journal of Robust and Nonlinear Control, vol. 6, no. 9-10, pp. 983-998, 1996.

[6] F. Wu and K. Dong, "Gain-scheduling control of LFT systems using parameter-dependent lyapunov functions," Automatica, vol. 42, no. 1, pp. 39-50, 2006.

[7] P. Apkarian and R. J. Adams, "Advanced gain-scheduling techniques for uncertain systems," IEEE Transactions on Control Systems Technology, vol. 6, no. 1, pp. 21-32, 1998.

[8] M. Chilali and P. Gahinet, " $\mathcal{H}_{\infty}$ design with pole placement constraints: an LMI approach," IEEE Transactions on Automatic Control, vol. 41, no. 3, pp. 358-367, 1996.
[9] G. Becker and A. Packard, "Robust performance of linear parametrically varying systems using parametricallydependent linear feedback," Systems and Control Letters, vol. 23, no. 3, pp. 205-215, 1994.

[10] A. S. Ghersin and R. S. Sánchez Peña, "LPV control of a 6 DOF vehicle," IEEE Transactions on Control Systems Technology, vol. 10, no. 6, pp. 883-887, 2002.

[11] C. Scherer, P. Gahinet, and M. Chilali, "Multiobjective outputfeedback control via LMI optimization," IEEE Transactions on Automatic Control, vol. 42, no. 7, pp. 896-911, 1997.

[12] A. S. Ghersin, Implementation of advanced linear parameter control techniques, Ph.D. thesis, Faculty of Engineering, University of Buenos Aires, Buenos Aires, Argentina, 2009.

[13] M. Noton, Spacecraft Navigation and Guidance, Springer, London, UK, 1996.

[14] J. Nielsen, Missile aerodynamics, NEAR, 1999.

[15] A. Elfving, L. Stagnaro, and A. Winton, "Smart-1: key technologies and autonomy implementations," Acta Astronautica, vol. 52, no. 2-6, pp. 475-486, 2003.

[16] M. Galassi, J. Davies, J. Theiler, et al., Gnu Scientific Library: Reference Manual, Network Theory Limited, Bristol, UK, 3rd edition, 2003.

[17] P. Gerum, "Xenomai-Implementing a RTOS emulation framework on GNU/Linux, 2004," http://www.xenomai.org/.

[18] T. Straumann, "Open source real-time operating systems overview," in Proceedings of the 8th International Conference on Accelerator and Large Experimental Physics Control Systems, pp. 27-30, 2001.

[19] P. Apkarian, "On the discretization of LMI-synthesized linear parameter-varying controllers," Automatica, vol. 33, no. 4, pp. 655-661, 1997. 

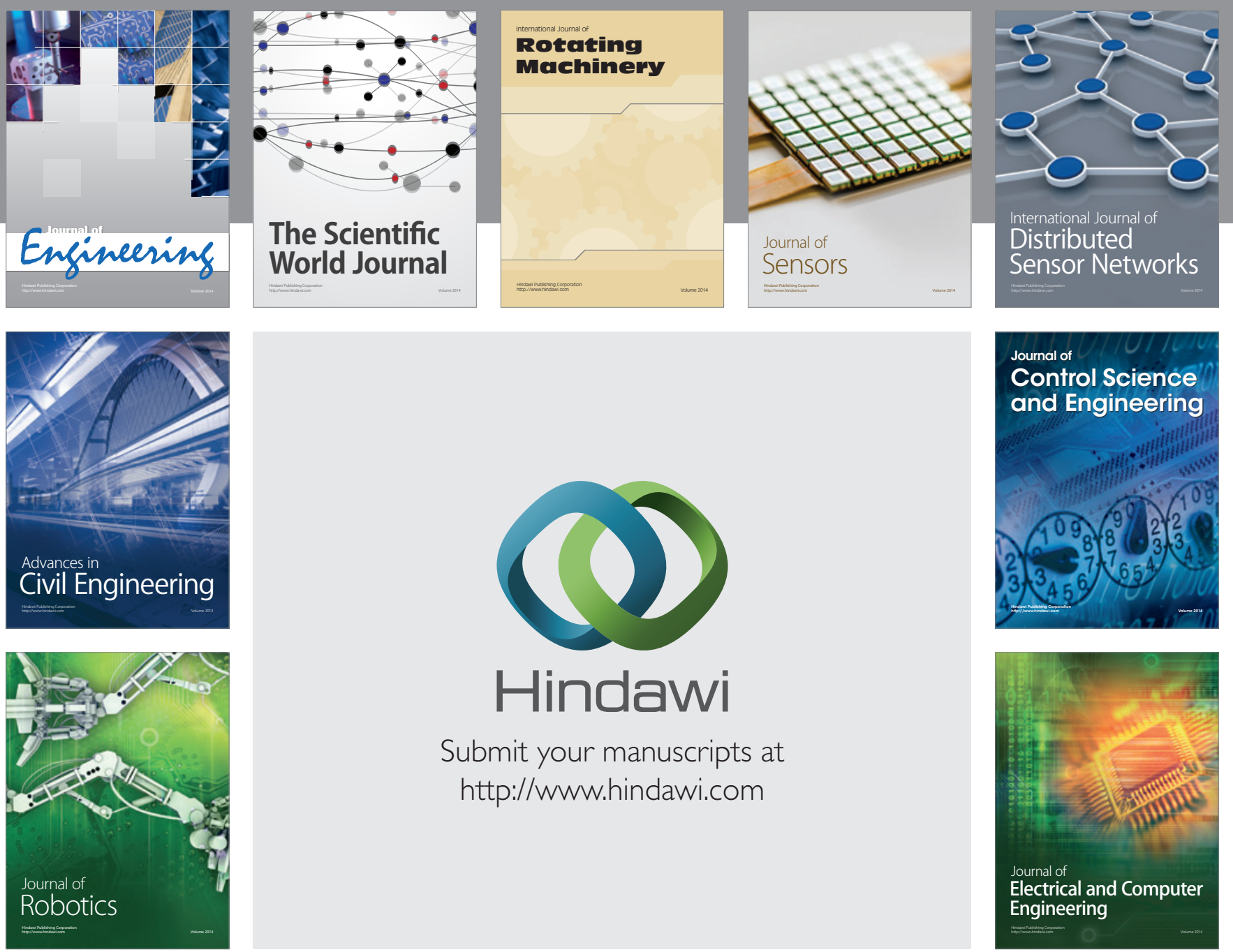

Submit your manuscripts at

http://www.hindawi.com
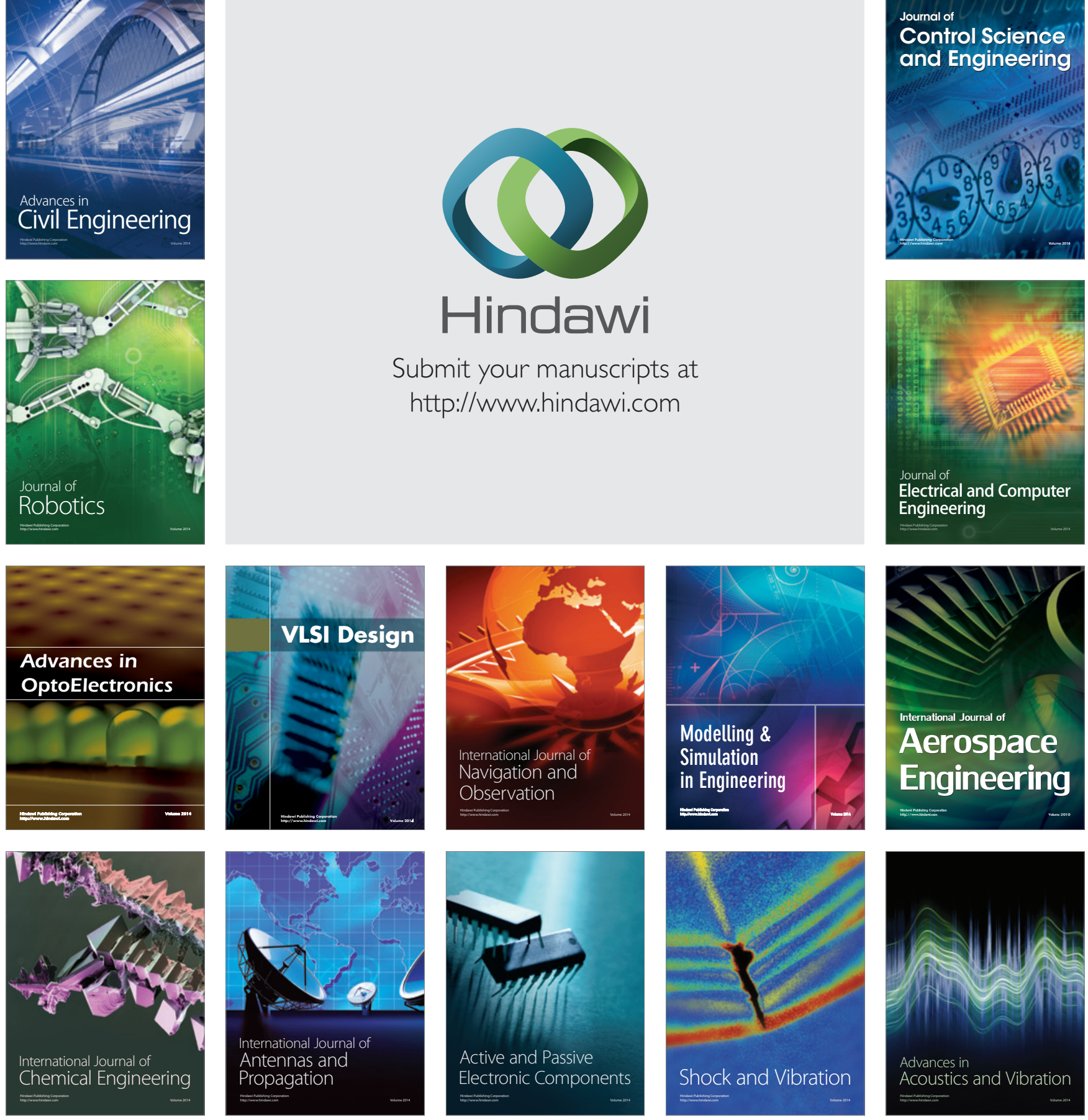